\title{
Withholding Antimalarials in Febrile Children Who Have a Negative Result for a Rapid Diagnostic Test
}

\author{
Valérie d'Acremont, ${ }^{1,3}$ Aggrey Malila, ${ }^{4}$ Ndeniria Swai, ${ }^{3}$ Robert Tillya, ${ }^{4}$ Judith Kahama-Maro, ${ }^{3}$ Christian Lengeler, ${ }^{1}$ \\ and Blaise Genton ${ }^{1,2,4}$
}

${ }^{1}$ Swiss Tropical and Public Health Institute, Basel, and ${ }^{2}$ Department of Ambulatory Care and Community Medicine, University of Lausanne, Lausanne, Switzerland; and ${ }^{3}$ City Medical Office of Health and ${ }^{4}$ Ifakara Health Institute, Dar es Salaam, Tanzania

\section{(See the editorial commentary by Björkman and Mårtensson, on pages 512-514.)}

Background. The availability of a rapid diagnostic test for malaria (RDTm) allows accurate diagnosis at all levels of health facilities. The objective of the present study was to evaluate the safety of withholding antimalarials in febrile children who have a negative test result.

Methods. We conducted a prospective 2-arm longitudinal study in areas of Tanzania that are moderately and highly endemic for malaria. Children with a history of fever were managed routinely by resident clinicians of 2 health facilities, except that no antimalarials were prescribed if the RDTm result was negative. Children were followed up at home on day 7. The main outcome was the occurrence of complications in children with negative RDT $m$ results; children with positive RDT $m$ results were followed up for the same outcomes for indirect comparison.

Results. One thousand children (median age, 24 months) were recruited. Six hundred three children $(60 \%)$ had a negative RDTm result. Five hundred seventy-three (97\%) of these children were cured on day 7. Forty-nine $(8 \%)$ of the children with negative RDTm results spontaneously visited the dispensary before day 7 , compared with $10(3 \%)$ of the children with positive RDTm results. All children who had negative initial results had negative results again when they were tested either at spontaneous attendance or on day 7 because they were not cured clinically, except for 3 who gave positive results on days 2, 4, and 7 respectively but who did not experience any complication. Four children who had negative initial results were admitted to the hospital subsequently, all with negative results for malaria tests upon admission. Two of them died, of causes other than malaria.

Conclusions. Not giving antimalarial drugs in febrile children who had a negative RDT $m$ result was safe, even in an area highly endemic for malaria. Our study provides evidence for treatment recommendations based on parasitological diagnosis in children $<5$ years old.

The number of episodes of fever among African children could be as high as 870 million per year [1]. Among children who reach outpatient clinics across the continent, a presumptive diagnosis of malaria is given in $30 \%-40 \%$ of these cases [2]. Malaria appears therefore to be the number one cause of fever, as well as the leading cause of mortality, in children in Sub-Saharan Africa [3]. In 2006, the World Health Organization (WHO) published the following treatment recommendation: "In areas of high stable malaria transmission,

Received 30 January 2010; accepted 22 April 2010; electronically published 19 July 2010.

Reprints or correspondence: Dr Valérie d'Acremont, Swiss Tropical and Public Health Institute, PO Box 4002, Basel, Switzerland (valerie.dacremont@unibas.ch).

Clinical Infectious Diseases 2010;51(5):506-511

(C) 2010 by the Infectious Diseases Society of America. All rights reserved. 1058-4838/2010/5105-0003\$15.00

DOI: $10.1086 / 655688$ the prior probability of fever in a child being caused by malaria is high. Children $<5$ years of age should therefore be treated on the basis of a clinical diagnosis of malaria. There is as yet no evidence to show that the benefits of parasitological diagnosis in this highly vulnerable group outweigh the risks of not treating false-negatives" [4]. Obviously, the prime reason for this recommendation of blanket antimalarial treatment is to save lives. It takes into account the lack of sensitivity and lack of specificity of the clinical diagnosis of malaria [5].

Although this recommendation was probably sound at the time of its formulation, it should now be reviewed because of the steady decrease in malaria transmission that is occurring all over Sub-Saharan Africa. This decrease is due to large-scale control $[6,7]$ and the availability of reliable, inexpensive, and easy-to-use malaria 
diagnostic tools [8]. One consequence of the falling transmission rates is that the blanket approach to treatment is not as safe as it was a decade ago, because the malaria-attributable fraction of fevers is now substantially lower and therefore the relative likelihood of missing other potentially fatal diseases has become higher [9]. To support a change to this recommendation, WHO looks for convincing evidence on the safety of treating children $<5$ years old who live in areas endemic for malaria on the basis of a malaria test result.

The few studies that have looked at the outcome of treating febrile children who live in areas endemic for malaria on the basis of a malaria laboratory test suggest that this strategy might be safe [10-12]. To our knowledge, our study is the first prospective study in an area that is highly endemic for malaria that took place under routine implementation conditions with the aim of evaluating the safety of withholding antimalarials in febrile children who have a negative result for a rapid diagnostic test for malaria (RDTm). Our primary interest was the clinical outcome of the children, namely the rate of complications and deaths, irrespective of any other consideration (eg, parasitemia). The RDT $m$ testing by health care workers in both of our study settings was found to be reliable in a previous study [13].

\section{METHODS}

\section{Study Areas}

This work is part of a larger project on the introduction of RDT $m$ in Tanzania and the evaluation of its impact on health outcomes, drug prescriptions, consultation processes, patient and health care worker satisfaction, and cost saving (IMALDIA: Understanding and Improving Malaria Diagnosis in Tanzania). The present study was performed first in an area of moderate endemicity and then in an area of high endemicity. This stepwise design was chosen to ensure the safety of the patients. If the strategy of withholding antimalarials in febrile children with a negative malaria test result were shown to be not safe in an area of moderate endemicity, the second part of the study in an area of high endemicity would be canceled.

Dar es Salaam. Buguruni Health Center of Ilala Municipality in the city center of Dar es Salaam, Tanzania, was chosen as a place of recruitment because of its characteristics typical of the urban setting. This area is moderately endemic for malaria, with parasite rates in the community at $\sim 1 \%-4 \%$ [14] and with only $5 \%-10 \%$ of febrile patients being parasitemic [15].

Signal. Signal is a remote village in the Kilombero Valley (south-central Tanzania). The local health facility was chosen because of the ready availability of RDT $m$, the high percentage of febrile patients having parasites ( $50 \%)$, and the sufficiently high average number of patients attending daily (30), compared with the characteristics of the other remote dispensaries in the Kilombero Valley. RDT $m$ had been introduced in 7 health facilities in the area 1 year previously, with a 1-day initial training of all health care workers, a 2-month follow-up of general performance, and a routine quality assurance of the RDTm (including lot testing at a WHO reference laboratory) every 3 months. No further training was conducted in the Signal health facility in the frame of the present study.

\section{Study Subjects}

Inclusion criteria were as follows:

- 1. Children were aged from 6 months to 10 years in Dar es Salaam and from 2 months to $<5$ years in Signal.

- 2. Children were attending the health facility for the first time for the present problem.

- 3. There was a history of fever in the previous 48 hours or axillary temperature of $\geqslant 37.5^{\circ} \mathrm{C}$ at the time of consultation.

- 4. There was an absence of severe illness that required specific treatment or referral.

- 5. The main complaint was neither injury nor trauma.

\section{Study Procedures}

Oral informed consent was obtained from the parent or guardian. Patients were managed at the discretion of the clinicians, except that the latter were asked to perform an RDTm (ParaHitf cassette; Span Diagnostics) and to not prescribe antimalarials if the result was negative. A blood slide was also prepared as back-up for retrospective investigation of complicated cases. Patients were asked to come back to the same health facility in case of any problem. In Dar es Salaam, parents and guardians were informed that they would be visited at home after 7 days ( \pm 1 day) to assess whether the child was cured. In Signal, we asked them to come back on day 7 for the same reason. If they had not returned on the due day before 2:00 PM, they were visited at home on the same or on the following day. At each home, the clinician would ask the parent or guardian about the status of the child (cured, not improved, or condition worsened) and whether they had gone to another health facility in the meantime.

If the child were ill between day 0 and day 7 or not cured on day 7 , a new consultation was performed by the usual clinician of the health facility, and the patient was tested again with either an RDTm (if the initial RDTm test result had been negative) or by microscopy (if the initial RDT $m$ test result had been positive). Patient management was again left to the discretion of the clinician, and antimalarials were again withheld if the test was negative. The child was then visited at home on day 14 to check his or her status. For admitted children, blood slides for malaria were read by 2 independent expert microscopists.

Children who had positive RDT $m$ results were treated with 
the recommended first-line antimalarial (artemether-lumefantrine [Coartem]) and were followed up in the same way as children who had negative RDT $m$ results. For obvious reasons, they did not represent a proper control group, because they represented a clinically very different group of children. Consequently, no tests of significance were performed to compare the 2 groups.

We calculated that a sample of 500 malaria-negative children was required to estimate an adverse event rate of $5 \%$ with a precision of $\pm 2 \%$. This risk was estimated on the basis of the sensitivity of routine RDTm (90\%) combined with a 50\% risk of complication (defined as hospital admission or death) due to delayed diagnosis and therefore absence of timely treatment.

The study protocol and related documents were approved by the Ethikkommission beider Basel (EKBB) in Switzerland and by the National Institute for Medical Research Review Board in Tanzania. The procedures followed were in accordance with the ethical standards of the responsible committee on human experimentation and with the Helsinki Declaration.

\section{RESULTS}

\section{Status at Inclusion}

In Buguruni health centre (Dar es Salaam), 300 children with a median age of 28 months (range, 3-120 months, with 259 [86\%] being $\leqslant 5$ years old) were included in May-June 2007. One hundred forty-three children (48\%) were female. All had a history of "homa," the Swahili word for fever, although this was the main complaint in only $205(68 \%)$ of the cases. The axillary temperature was $\geqslant 37.5^{\circ} \mathrm{C}$ in $118(39 \%)$ of the children. At inclusion, 41 (14\%) of the 300 children had a positive RDT $m$ result. All 41 children with positive results were treated with an antimalarial: 35 (85\%) received artemether-lumefantrine and the rest received oral quinine. Thirteen children with positive results (32\%) also received an antibiotic. Except for one child, all children with negative results received an antibiotic (cotrimoxazole, amoxicillin, and injectable penicillin were mostly used).

In the Signal dispensary (Kilombero), 700 children with a median age of 24 months (range, 2-59 months) were included from September 2008 through February 2009. Three hundred forty-one (49\%) were females. They all had a history of homa, which was the main complaint in $604(86 \%)$ of the cases. The axillary temperature was $\geqslant 37.5^{\circ} \mathrm{C}$ in $404(58 \%)$ of the children. At inclusion, 356 (51\%) of the 700 children had a positive RDT $m$ result. All children with positive results were treated with an antimalarial: 342 (96\%) received artemisinin-lumefantrine and the rest received injectable or oral quinine. Sixty-four (18\%) of the 356 children with positive results also received an antibiotic. Three hundred twelve (91\%) of the 344 children with negative results received an antibiotic.

\section{Spontaneous Attendance before Day 7}

In total, $59(5.9 \%)$ of the 1000 children consulted spontaneously before day 7 , the majority of them (45 [76\%]) visiting the same facility that they visited for the initial consultation. Among the 603 children who had a negative RDTm result at inclusion, 49 (8\%) (representing 31 [12\%] of 259 in Dar es Salaam and 18 [5\%] of 344 in Signal) attended before day 7 (median, 3 days after inclusion), with persisting fever in 37 children (despite antibiotics prescribed initially for 35 of them). Among the 397 children who had a positive RDTm result at inclusion, 10 (3\%) attended spontaneously before day 7 (median, 4 days after inclusion), with persisting fever in 5 children ( 4 of them had received an antibiotic at inclusion in addition to the antimalarial) (Figure 1).

\section{Outcome on Day 7 and Day 14}

Thirteen (1.3\%) of the 1000 children included were lost to followup, either because they had moved or the address was not sufficiently precise. Among the 591 children who had a negative RDT $m$ result, 573 (97\%) were cured on day 7. For the remaining 18 children, 15 were examined before or on day 7 because of fever, 2 because of diarrhea, and 1 because of a headache. Of these 18 children, 14 were cured on day 14, 2 died (see below), and 2 were lost to follow-up after day 7 (both had negative RDTm results on day 7). Among the 396 children who had a positive RDT $m$ result, 387 (98\%) were cured on day 7 (38 [95\%] of 40 in Dar es Salaam and 349 [98\%] of 356 in Signal). Eight children were still febrile (all had a negative blood slide results), and 1 had asthma. All these children were cured on day 14 (1 was lost to follow-up after day 7) (Figure 1).

\section{Complications}

Of the 603 children who had a negative RDTm result at inclusion, 4 ( 1 in Dar es Salaam and 3 in Signal) were hospitalized: 1 on day 2 because of severe sepsis with exanthema, 1 on day 4 because of severe pneumonia, 1 on day 7 because of gastroenteritis with severe dehydration, and 1 on day 7 because of severe anemia without fever. These 4 children had an RDTm and an expert microscopy for malaria performed upon admission at the hospital and all gave negative results. Two children died ( 1 with severe sepsis died on day 6 , and 1 with severe pneumonia died on day 8). Among the 397 children who had a positive RDT $m$ result at inclusion, 1 was hospitalized on day 7 , because pneumonia had not responded to the first-line intravenous antibiotic treatment (Figure 1).

\section{Subsequent RDTm Results of the Children with Negative Initial Results Who Consulted before Day 7 or Who Were Not Cured on Day 7}

Among the 603 children who had negative RDT $m$ results at inclusion, 55 consulted spontaneously before day 7 or were not 


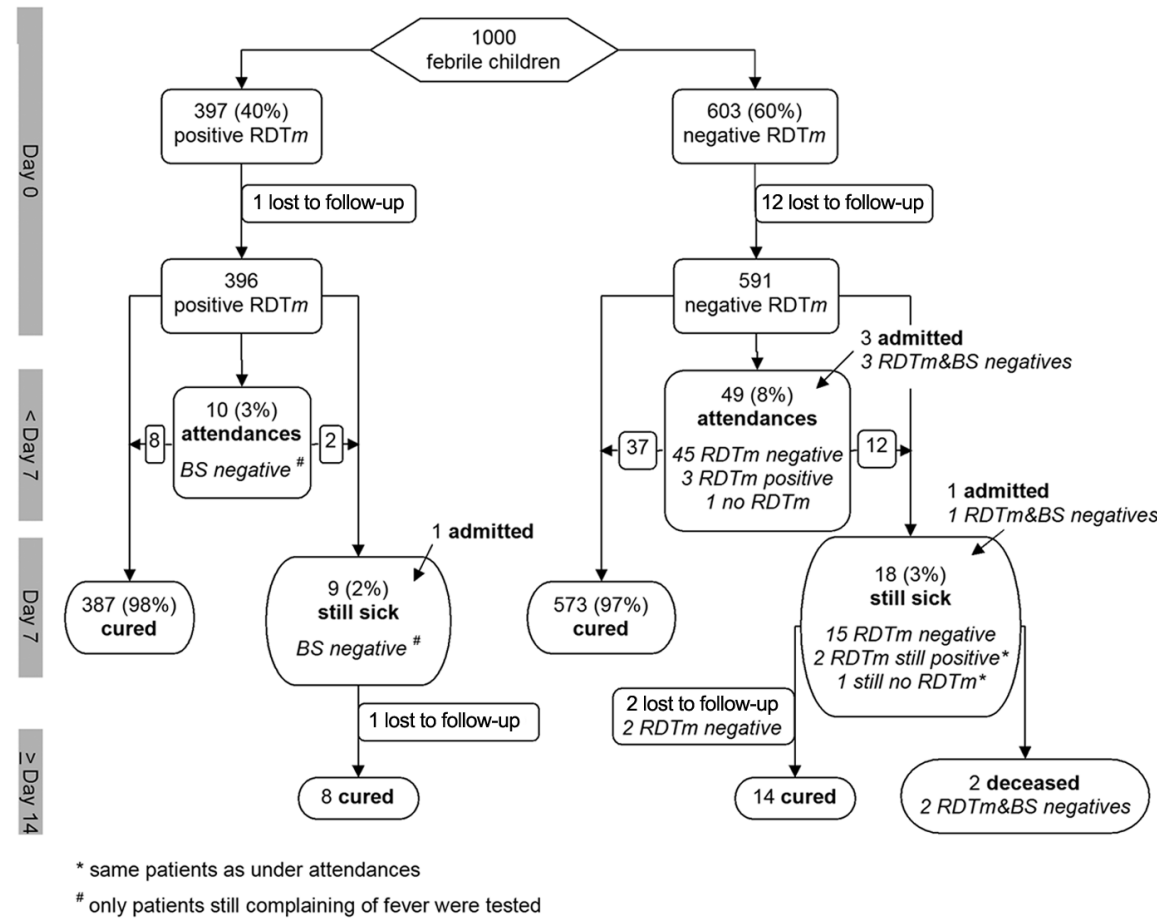

Figure 1. Flow chart of patients in a study of withholding antimalarials in febrile children who have a negative result for a rapid diagnostic test in Tanzania. BS, [result of] blood slide for malaria; RDTm, [result of] rapid diagnostic test for malaria.

cured on day 7 when we visited them at home. Of these 55 children, 51 had negative RDT $m$ results; one had positive results on day 4 and two on day 7 (all living in Signal, the area of high endemicity), and one could not be tested but recovered on day 14 after receiving erythromycin prescribed in another health facility. Among the 3 with newly positive results, 1 child was likely to have had coincidental low-density parasitemia. Indeed, he had positive results on day 4 and did not respond to the antimalarial treatment given on that day, but he responded to an antibiotic given on day 7. Another child had dysuria at inclusion, received amoxicillin, and recovered, but he became feverish again at day 5 with dysuria; he had positive test results at day 7 and was treated with intramuscular quinine and cotrimoxazole and was cured on day 14 . He had either a coincidental or a new infection. The last child had vomiting on day 0 and was treated with cotrimoxazole; then, because of persisting fever, he attended another dispensary on day 2 where he was not tested for malaria but received oral quinine. He was cured on day 7 but had positive results by RDTm. He might have experienced a malaria episode that was not diagnosed on day 0 because of clinical symptoms that appeared before patent parasitemia.

\section{DISCUSSION}

To our knowledge, our study is the first to provide strong evidence that withholding antimalarial treatment in children with fever who have a negative RDT $m$ result in a setting highly endemic for malaria is safe and does not expose the child to an increased risk of complication or death. Because the present study was conducted with programmatic conditions that were reasonably close to routine, we believe that the results are applicable more generally.

\section{Complications or Deaths}

In this cohort of 1000 feverish children, 60\% had a negative $\mathrm{RDT} m$ result at inclusion and were not treated with an antimalarial. We did not observe any complication or death due to malaria among these children. In total, 4 children $(0.7 \%)$ were admitted to the hospital and $2(0.4 \%)$ died; malaria test results on admission were negative for all 4 . Our study provides strong evidence that the strategy is safe, irrespective of the level of endemicity of malaria. To our knowledge, the only other study with a follow-up of fever episodes in untreated children with negative test results (tested by expert microscopy rather than by RDTm) was the study by Njama-Meya et al [10] in Kampala, Uganda. That study, designed primarily to assess the efficacy of antimalarial drugs, was performed in a highly controlled setting and included children with danger signs. No complication or death due to the withholding of antimalarial treatment in children with negative test results occurred.

Although the absence of a proper control group did not allow us to know the usual rate of complications in the absence of 
any reliable malaria test, the lack of admissions and/or deaths due to malaria following RDTm use certainly suggested that this approach is safe. One $(0.3 \%)$ of the 397 children in the malaria group and $4(0.7 \%)$ of the 603 children in the nonmalaria group were admitted after first consultation. There was no death in the malaria group and 2 deaths in the nonmalaria group. We know that these complications in nonmalaria patients were not due to undetected malaria parasites, because we performed $\geqslant 3$ malaria tests on each of these children and none had positive results.

On the basis of 2 previous studies on severe malaria conducted in Tanzania, we knew that the rate of adverse outcomes is higher in nonmalaria patients, compared with the rate in malaria patients, because diseases such as pneumonia, typhoid, and meningitis have a fatality rate at least as high as that of malaria and because severely ill febrile patients are often misdiagnosed as having malaria and therefore do not receive appropriate antibiotics $[16,17]$. Such a trend was also found in our study, which included less severe cases. Therefore, accurate diagnosis is essential even at the outpatient level to prevent the further occurrence of complications and deaths. This is especially true now, because of the regular decrease in malaria transmission in many settings of endemicity $[6,18,19]$ and therefore the relative increase of fevers due to other treatable causes in many places in Africa.

\section{Outcome on Day 7}

In our study, the percentage of children not cured on day 7 was very low in both groups ( $2 \%$ in the malaria group versus $3 \%$ in the nonmalaria group). The 9 malaria patients who were still sick after 1 week all had a negative result for a follow-up blood slide test. Therefore, it is very likely that they were suffering from another fever cause, with parasitemia being coincidental. For the nonmalaria cases, 17 of the 18 children who were not cured on day 7 had received an antibiotic, which suggests that they had either a viral disease with prolonged fever or a bacterial disease resistant to the first-line antibiotic. In these 2 areas, we know that nonmalaria fevers are composed of $56 \%$ acute respiratory infections, $10 \%$ gastroenteritis, $6 \%$ urinary infections, $3 \%$ typhoid, $3 \%$ other documented infections, and 22\% fever of unknown origin. Furthermore, $68 \%$ of acute respiratory infections and $31 \%$ of gastroenteritis episodes are associated with documented viruses (d'Acremont et al, unpublished data, 2010) that obviously do not respond to antibiotics and that therefore tend to persist longer than malaria episodes treated with an effective drug. For nonmalaria fevers due to bacteria, the high rate of resistance to first-line antibiotics in Tanzania also leads to prolonged episodes [20].

\section{Outcome of Children with Negative Initial Results and Positive Subsequent Results}

There are several reasons for which a malaria test can yield negative initial results and positive subsequent results within a few days: (1) coincidental low-density malaria fluctuating around the threshold of detection of a malaria test; (2) symptoms and signs appearing before parasites can be detected in the blood by conventional tests, especially in nonimmune infants or young children; (3) new infections; and (4) failure of the test (negative test result in the presence of normal density parasitemia). Coincidental or new infection is the most likely explanation for 2 of the 3 patients with negative initial results and positive subsequent results. In an area of high endemicity, it is expected to have some children presenting with new parasitemia every day. We found in our sample of children from Signal an incidence of malaria in the 7 days after a negative test result of 0.45 episodes per child per year, which is lower than expected. This rate is close to the one reported by NjamaMeya et al [10] (0.42 episodes per child per year) and found by using microscopy instead of RDTm. It is also similar to the incidence of malaria episodes in infants ( 0.43 episodes per infant per year) found by using passive-case detection in the main town of Kilombero District in the year 2000 [21]. Failure of the RDTm to detect parasitemia of a density high enough to be detected by conventional test probably did not occur in our study, because all 3 patients who had negative RDT $m$ results at inclusion also had negative results by expert microscopy. In the study by Njama-Meya et al [10], there were 4 children with negative results by initial expert microscopy but positive results by subsequent expert microscopy. False-negative results do exist with RDTm but possibly at a lower rate than with expert microscopy, as demonstrated by Ochola et al [22] and Bell et al [23].

\section{CONCLUSIONS}

In 2 Tanzanian settings ( 1 urban and 1 rural), the strategy of RDT $m$-based diagnosis and treatment proved to be safe, with no case that was missed by a negative initial $\mathrm{RDT} m$ result ending in complication or death. The sample size of our study allowed us to say with $95 \%$ confidence that the probability of such an unfavorable event was between $0 \%$ and $0.5 \%$ (rule of 3) [24]. Although our study does not provide the definite answer in this matter, it contributes important evidence toward a change in policy in malaria diagnosis and treatment and thus toward a better management of fever in countries endemic for malaria.

\section{Acknowledgments}

We thank the clinicians, nurses, laboratory staff, and community workers at Buguruni Health Center and at Signal Dispensary who participated in 
the study, particularly Charles Kasmil, the clinician in charge of Signal Dispensary. We are grateful to Gerumana Mpamwa from the City Medical Office of Health, Dar es Salaam, Tanzania, for data entry and logistic support. We also thank the inhabitants of Buguruni and Vingunguti wards and the villages around Signal for participating.

Financial support. Swiss National Science Foundation (grant 3270B0109696 to V.d.A. and to the project).

Potential conflicts of interests. All authors: no conflicts.

\section{References}

1. Snow RW, Eckert E, Teklehaimanot A. Estimating the needs for artesunate-based combination therapy for malaria case-management in Africa. Trends Parasitol 2003; 19:363-369.

2. Chima RI, Goodman CA, Mills A. The economic impact of malaria in Africa: a critical review of the evidence. Health Policy (New York) 2003; 63:17-36.

3. Rowe AK, Rowe SY, Snow RW, et al. The burden of malaria mortality among African children in the year 2000. Int J Epidemiol 2006; 35: 691-704.

4. World Health Organization. Guidelines for the treatment of malaria. Geneva, Switzerland: World Health Organization, 2006.

5. Chandramohan D, Jaffar S, Greenwood B. Use of clinical algorithms for diagnosing malaria. Trop Med Int Health 2002; 7:45-52.

6. World Health Organization. Impact of long-lasting insecticidal treated nets (LLINs) and artemisinin-based combination therapies (ACTs) measured using surveillance data, in four African countries. Geneva, Switzerland: World Health Organization, 2008.

7. Guerra CA, Gikandi PW, Tatem AJ, et al. The limits and intensity of Plasmodium falciparum transmission: implications for malaria control and elimination worldwide. PLoS Med 2008; 5:e38.

8. Drakeley C, Reyburn H. Out with the old, in with the new: the utility of rapid diagnostic tests for malaria diagnosis in Africa. Trans R Soc Trop Med Hyg 2009; 103:333-337.

9. d'Acremont V, Lengeler C, Mshinda H, Mtasiwa D, Tanner M, Genton B. Time to move from presumptive malaria treatment to laboratoryconfirmed diagnosis and treatment in African children with fever. PLoS Med 2009; 6:e252.

10. Njama-Meya D, Clark TD, Nzarubara B, Staedke S, Kamya MR, Dorsey G. Treatment of malaria restricted to laboratory-confirmed cases: a prospective cohort study in Ugandan children. Malar J 2007; 6:7.

11. Ngasala B, Mubi M, Warsame M, et al. Impact of training in clinical and microscopy diagnosis of childhood malaria on antimalarial drug prescription and health outcome at primary health care level in Tanzania: a randomized controlled trial. Malar J 2008; 7:199.

12. Msellem MI, Mårtensson A, Rotllant G, et al. Influence of rapid malaria diagnostic tests on treatment and health outcome in fever patients, Zanzibar: a crossover validation study. PLoS Med 2009; 6:e1000070.
13. Kahama-Maro J, d'Acremont V, Mtasiwa D, Genton B, Lengeler C. Low quality of routine microscopy for malaria at different health system levels in Dar es Salaam: rapid diagnostic tests should also be implemented in hospitals and urban settings. In: Program and abstracts of the 57th Annual Meeting of the American Society of Tropical Medicine and Hygiene. Deerfield, IL: American Society of Tropical Medicine and Hygiene, 2008. Abstract 394.

14. Wang S, Lengeler C, Mtasiwa D, et al. Rapid Urban Malaria Appraisal (RUMA) II: epidemiology of urban malaria in Dar es Salaam (Tanzania). Malar J 2006; 5:28.

15. d'Acremont V, Kahama-Maro J, Mtasiwa D, Genton B, Lengeler C. Massive reduction of antimalarial prescriptions after Rapid Diagnostic Tests implementation in Dar es Salaam, Tanzania. In: Program and abstracts of the 57th Annual Meeting of the American Society of Tropical Medicine and Hygiene. Deerfield, IL: American Society of Tropical Medicine and Hygiene, 2008. Abstract 577.

16. Makani J, Matuja W, Liyombo E, Snow RW, Marsh K, Warrell DA. Admission diagnosis of cerebral malaria in adults in an endemic area of Tanzania: implications and clinical description. QJM 2003; 96:355362.

17. Reyburn H, Mbatia R, Drakeley C, et al. Overdiagnosis of malaria in patients with severe febrile illness in Tanzania: a prospective study. BMJ 2004; 329:1212.

18. Ceesay SJ, Casals-Pascual C, Erskine J, et al. Changes in malaria indices between 1999 and 2007 in the Gambia: a retrospective analysis. Lancet 2008; 372:1545-1554.

19. O'Meara WP, Bejon P, Mwangi TW, et al. Effect of a fall in malaria transmission on morbidity and mortality in Kilifi, Kenya. Lancet 2008; 372:1555-1562.

20. Blomberg B, Manji KP, Urassa WK, et al. Antimicrobial resistance predicts death in Tanzanian children with bloodstream infections: a prospective cohort study. BMC Infect Dis 2007; 7:43.

21. Schellenberg D, Menendez C, Aponte J, et al. The changing epidemiology of malaria in Ifakara Town, southern Tanzania. Trop Med Int Health 2004; 9:68-76.

22. Ochola LB, Vounatsou P, Smith T, Mabaso MLH, Newton CRJC. The reliability of diagnostic techniques in the diagnosis and management of malaria in the absence of a gold standard. Lancet Infect Dis 2006; 6:582-588.

23. Bell DR, Wilson DW, Martin LB. False-positive results of a Plasmodium falciparum histidine-rich protein 2-detecting malaria rapid diagnostic test due to high sensitivity in a community with fluctuating low parasite density. Am J Trop Med Hyg 2005; 73:199-203.

24. Eypasch E, Lefering R, Kum CK, Troidl H. Probability of adverse events that have not yet occurred: a statistical reminder. BMJ 1995; 311:619620. 